Meta

Journal des traducteurs

Translators' Journal

\title{
Terminologie de l'assurance-vie
}

\section{Jean-Paul de Grandpré}

Volume 19, numéro 3, septembre 1974

URI : https://id.erudit.org/iderudit/002963ar

DOI : https://doi.org/10.7202/002963ar

Aller au sommaire du numéro

Éditeur(s)

Les Presses de l'Université de Montréal

ISSN

0026-0452 (imprimé)

1492-1421 (numérique)

Découvrir la revue

Citer cet article

de Grandpré, J.-P. (1974). Terminologie de l'assurance-vie. Meta, 19(3), 150-153.

https://doi.org/10.7202/002963ar

Ce document est protégé par la loi sur le droit d'auteur. L'utilisation des services d'Érudit (y compris la reproduction) est assujettie à sa politique d'utilisation que vous pouvez consulter en ligne.

https://apropos.erudit.org/fr/usagers/politique-dutilisation/ 


\section{TERMINOLOGIE DE L'ASSURANCE-VIE}

Carences terminologiques et anarchie aux niveaux du vocabulaire et de la syntaxe ont caractérisé depuis toujours la langue technique utilisée au Québec. Une pareille affirmation n'étonne maintenant personne. Il y a dix ans, elle aurait choqué. C'est que depuis il s'est produit un réveil de la conscience linguistique et parallèlement l'amorce d'un redressement. 
Dans les quelques lignes qui suivent j'évoquerai le cheminement de cette prise de conscience dans un secteur particulier, celui de l'assurance sur la vie, et je tenterai d'évaluer l'un des projets envisagés : la confection d'un vocabulaire exhaustif.

En 1967 paraissait le Vocabulaire bilingue des assurances sur la vie, le troisième de la série des cahiers que publie l'Office de la langue française. L'accueil qui lui fut fait dans la presse et celui des praticiens de la langue ont prouvé l'opportunité d'une telle publication.

Une certaine perplexité se manifesta d'abord dans l'industrie. Mais l'hésitation fut de courte durée. On apprit à apprécier cet instrument de travail, si incomplet fût-il. Les dirigeants de nombreuses sociétés d'assurance (plusieurs m'en ont fait part par écrit) reconnurent que cet ouvrage permettait une amélioration considérable de la communication, entre employés d'abord, puis entre les sociétés et organismes professionnels et les services administratifs féféraux et provinciaux.

On entrevit, pour la première fois peut-être dans l'industrie, la possibilité d'utiliser une langue sûre, d'ordonner le fouillis de termes abusifs et souvent dangereux utilisés jusque-là. Chez les anglophones, l'idée de pouvoir atténuer le cauchemar de la traduction commençait à poindre.

Il s'ensuivit un accord des volontés et des résultats positifs qui devaient s'échelonner sur quelques années.

Dès 1967, les membres du bureau de 1'Association provinciale des assureursvie décidaient d'adopter la terminologie pratiquée dans les autres pays francophones. Cette décision entraînait du même coup l'adhésion de l'Association canadienne elle-même, car les producteurs parlant le français sont en très grande majorité regroupés au Québec.

Jusque-là, les acquiescements avaient été surtout le fait des éléments de base : les producteurs et les directeurs d'agence, ceux-là mêmes pour qui la langue est l'instrument par excellence, celui qui les met en mesure par la clarté des exposés, par la vigueur des argumentations, de recruter la clientèle. Leur enthousiasme permettait d'espérer davantage : un assentiment en haut lieu, celui de l'Association des compagnies d'assurance-vie du Canada.

Cette approbation ne devait guère tarder non plus. En 1971, cette association, qui regroupe la plupart des sociétés Vie faisant des affaires au Canada, décrétait la normalisation de la langue de sa discipline.

L'unanimité était enfin réalisée. Le reste allait de soi. Moins d'un an après, un accord intervenait entre l'Office de la langue française et cette association qui prévoyait la confection de fiches normatives et leur distribution.

Ces fiches, que je rédige avec M. Lucien Forgues, sont soumises à un comité groupant des représentants des sociétés, des assureurs, d'un organisme technique et de l'Office ${ }^{1}$. Le comité de rédaction n'a eu qu'à se féliciter de l'à-propos des

1. Il s'agit de M. L.-P. Béguin, responsable à l'Office du secteur des assurances. M. Béguin a publié en 1972 un Vocabulaire correctif des assurances (cahier $\mathrm{n}^{\circ} 16$ ). Ce travail qui comprend un assez grand nombre d'expressions de langue courante intègre les rubriques du cahier $\mathrm{n}^{\circ} 3$, présentées par le biais de la forme fautive. 
quelques observations faites sur les fiches présentées. Les livraisons, qui sont commencées, se poursuivront désormais régulièrement.

Voilà pour les antécédents.

Ce qui reste à faire (je ne parle pas de la rédaction proprement dite qui m'est facilitée par la formidable documentation de M. Forgues et sa collaboration si efficace, ponctuelle et éclairée) incombe aux usagers : traducteurs et rédacteurs.

Ils auront fort à faire si nous voulons qu'un jour techniciens et praticiens de l'assurance utilisent des ouvrages pensés en français (ou à tout le moins bien traduits) qui les mettent à même d'apprendre bien, d'apprendre vite, de s'exprimer, de créer.

Mais le gros de l'effort reposera dans l'immédiat sur les traducteurs qui travaillent dans des entreprises dont la direction semble encore ignorer la rentabilité de la bonne langue. Sans doute ces collègues isolés des marches lointaines de la francophonie auront-ils un jour l'appui, par le truchement d'une critique éclairée, de la clientèle québécoise.

À ceux-là, et aux autres plus fortunés, je conseillerais d'abord l'acquisition d'une documentation d'assurance et la lecture des bons auteurs français. Les Assurances terrestres en droit français, de MM. Picard et Besson, est un ouvrage que tous les traducteurs d'assurance devraient posséder et méditer.

On trouvera également d'autres éléments de bibliographie dans les articles que j'ai déjà fait paraître dans la présente revue ${ }^{2}$. Je rappellerai ici que ces articles exposent quelques principes toujours valables dans l'exercice du métier. J'y ai traité de certains des termes qui sont à la base même des opérations d'assurance : la garantie, le règlement, et de quelques idiotismes propres à apporter aux traductions un peu de la concision française.

Je dirai maintenant un mot des fiches. Elles sont, somme toute, le résultat d'une assez longue expérience en traduction. Elles sont de plus rédigées (en ce qui me concerne) par un ancien professeur. D'où un certain souci pédagogique dans la présentation.

Les rubriques sont réparties en sept catégories, celles adoptées par l'A.F.N.O.R. :

01. Termes généraux - Assureurs - Assurés

02. Catégories d'assurance

03. La garantie et ses modalités

04. Primes - Commissions

05. Sinistres - Indemnités

06. Contrats d'assurance et gestion des contrats

07. Gestion administrative et financière des entreprises d'assurance et règlementation auxquelles nous en avons ajouté une huitième, locutions particulières, où se retrouvent des faits de langue n'appartenant pas nécessairement à l'assurance, mais dont le traitement s'impose au Québec.

2. Meta, vol. $11, \mathrm{n}^{\text {os }} 2$ et 3 ; vol. 12 , nos 2 et $4 ;$ vol. 14 , no 3 . 
Chaque fiche donne, en plus des rubriques anglaise et française, une définition dans les deux langues du terme étudié. C'est là un élément de motivation que mon collègue et moi avons cru indispensable. À ces définitions viennent s'ajouter des exemples, parfois nombreux. Ces citations, en plus de confirmer la justesse du terme ont une valeur de preuve, une valeur apologétique dirais-je : le lecteur aura compris qu'elles visent les employeurs incrédules ou ceux pour lesquels il importe de refaire l'image de la langue. Des renvois permettent des recoupements utiles et, par des simultanéités d'éclairage, l'approfondissement des termes rapprochés.

Ce lexique vise à renseigner, mais aussi à enseigner.

Les niveaux de langue y sont souvent précisés. Il faut savoir par exemple, que l'expression faire souscrire employée dans un traité devient vendre, dans une notice destinée à un simple apporteur; à un niveau familier, police d'assurance sur la vie s'abrège en police Vie; société mutuelle d'assurance contre les accidents en mutuelle Accidents.

Les extensions de sens, tellement craintes dans notre pays linguistiquement timoré, sont encouragées. Tarif, qui désigne le taux de prime applicable à tel capital proposé, s'entend aussi du livre où sont consignés tous les taux de prime, même s'ils sont accompagnés de longs textes précisant les modalités d'application (rate book : tarif, et non livre de taux).

La peur des homonymies et des rapprochements «scabreux » ou « saugrenus 》 est systématiquement combattue : nous parlons de producteurs, de combinaisons, malgré ces subtils préjugés qui, jusqu'à maintenant, avaient impitoyablement banni ces termes de l'usage.

Voilà donc en termes bien imparfaits et hâtifs quelques-uns des avantages de ce nouveau lexique.

Il reste que par-delà l'étude des terminologies, le traducteur doit acquérir une vaste culture. Il doit notamment pouvoir, après une minutieuse analyse du contenu et de l'esprit d'un texte, en faire une synthèse intégrale et concise dans laquelle la juxtaposition des idées évitera la lourdeur des subordonnées, et l'emploi du mot juste, l'orgie des épithètes.

Disons en guise de conclusion qu'un bon répertoire de termes utilisés par un esprit cultivé permet de bien dire en dix lignes ce qu'on ne réussit pas toujours à mal dire en vingt lignes. Puissent les dirigeants des sociétés d'assurance, par l'effet du vocabulaire qui fait l'objet de ces quelques considérations, encore mieux rentabiliser et rémunérer les capitaux qui leur sont confiés par les assurés.

JeAn-PAul de Grandpré 